\title{
La co-conception en partenariat de systèmes agricoles innovants
}

\author{
Eric Vall ${ }^{1, *}$, Eduardo Chia $^{2}$, Mélanie Blanchard ${ }^{1,3}$, Mahamoudou Koutou ${ }^{3}$, Kalifa Coulibaly ${ }^{3,4}$ \\ et Nadine Andrieu ${ }^{5,6}$ \\ ${ }^{1}$ CIRAD, UMR Selmet, TA C-112/A, Campus International de Baillarguet, 34098 Montpellier Cedex 5, France \\ 2 INRA, UMR Innovation, Place Viala, 34060 Montpellier, France \\ 3 CIRDES, Urpan, BP 454, Bobo-Dioulasso, Burkina Faso \\ ${ }^{4}$ UPB, IDR, Bobo-Dioulasso, Burkina Faso \\ 5 CIRAD, UMR Innovation, TA C-85/15, 34398 Montpellier Cedex 5, France \\ ${ }^{6}$ CIAT, Km 17, Recta Cali-Palmira, Valle Del Cauca, Cali, Colombie
}

\begin{abstract}
Résumé - La co-conception de systèmes agricoles innovants est une piste prometteuse pour répondre au défi de l'innovation, notamment pour les exploitations agricoles familiales africaines confrontées à de multiples changements. Mais il faut penser à la place et aux rôles tenus par de multiples acteurs (agriculteurs, conseillers, chercheurs) pour produire les changements souhaités par toutes les parties, et donc réfléchir à la question du partenariat dans le processus. Cet article présente la démarche de conception en partenariat de systèmes agricoles innovants (CPSAI) élaborée entre 2005 et 2015 dans l'ouest du Burkina Faso, notamment sur la gestion de la fumure organique des cultures. La CPSAI s'appuie sur un dispositif partenarial formalisé et sur une démarche progressive en trois phases itératives : (1) exploration et formalisation du partenariat, (2) conceptions et test des options de changement, (3) bilan et désengagement. Les résultats obtenus montrent que l'innovation est plus efficace et durable quand elle est le produit d'un travail partenarial.
\end{abstract}

Mots clés : innovation / recherche en partenariat / polyculture-élevage / Afrique de l'Ouest

\begin{abstract}
Participatory design of innovative farming systems. Co-design of innovative agricultural systems is a promising way to meet the challenge of innovation, particularly for African family farms facing multiple changes. But we must think of the place and role of multiple stakeholders (farmers, advisors, researchers) to produce the desired change by all parties, so consider the issue of partnership in the process. This paper presents the process for the design of innovative farming systems (PDIFS) developed between 2005 and 2015 in western Burkina Faso, including the management of manure. PDIFS uses a formalized partnership system and a progressive approach in three iterative phases: (1) exploration and formalization of partnership; (2) co-designs and testing options for change; (3) review and disengagement. The results show that innovation is more effective and sustainable when it is the product of a collective and partnership work.
\end{abstract}

Keywords: innovation / research in partnership / mixed farming / West Africa

\section{Introduction}

La co-conception de systèmes agricoles innovants est devenue une voie prometteuse, en agriculture, pour élaborer des propositions en adéquation technique, économique et organisationnelle avec les attentes et les possibilités des agriculteurs (Anadon, 2007). C'est le cas notamment en Afrique de l'Ouest et du Centre où les agriculteurs qui cherchent à innover sont confrontés à de multiples incertitudes (croissance démographique, aléa pluviométrique, volatilité des prix agricoles, désengagement des États, etc.) et à de nouvelles préoccupations environnementales (Adekunle et al., 2012).

$\overline{\text { *Auteur de correspondance }}$ : eric.vall@cirad.fr
L'objectif de la co-conception est de générer avec ces derniers des connaissances et des apprentissages qui déboucheront sur de nouveaux produits, de nouvelles technologies, de nouvelles organisations. Concevoir avec les acteurs des systèmes agricoles innovants est à l'évidence aujourd'hui un enjeu de recherche essentiel qui génère de nombreux travaux tant au Nord qu'au Sud, sur la co-construction du changement (Adekunle, 2009 ; Chia et Dedieu, 2002 ; Gouttenoire et al., 2014 ; Mikolasek et al., 2009 ; Vall et Chia, 2014).

La conception réglée, visant une amélioration de technologies existantes, en s'appuyant sur des objectifs prédéfinis, des compétences, des méthodes, des procédures d'évaluation standardisées, montre des limites dans un environnement incertain et changeant (Le Masson et al., 2006). La conception innovante, visant à satisfaire des attentes nouvelles, non 
spécifiées au départ, et se précisant à mesure que l'objet conçu prend forme, est plus adaptée. Ce dernier régime de conception a été développé en agriculture suivant deux branches. L'approche " de novo » privilégie l'invention de systèmes en rupture avec l'existant et la production de scénarios permettant d'explorer une large gamme de futurs possibles mettant en jeu des changements profonds. Cette approche « de novo » peut servir d'horizon à la conception « pas à pas » où l'on cherche surtout à organiser le changement en s'appuyant sur des boucles d'apprentissages progressifs. Dans l'approche "pas à pas », le producteur met au point son nouveau système progressivement (innovation incrémentale), en même temps qu'il apprend à le piloter, se convainc de son intérêt, et réorganise son travail et ses moyens de production (Dedieu et al., 2011 ; Meynard et Dourmad, 2014 ; Meynard et al., 2012). Mais les modalités de participation des acteurs de terrain (comment les mobiliser ? Distribuer les rôles ? Gérer les attentes ? Expérimenter avec eux ? Évaluer les résultats ?) et les effets produits par la participation en conception « pas à pas » restent peu documentés.

Au Burkina Faso, nous étions confrontés au départ à des acteurs ruraux (agriculteurs, conseillers agricoles...), demandeurs de changement et réunis par la volonté d'améliorer durablement les performances des systèmes agricoles, mais pourtant divisés sur les voies à suivre. Pour gérer cette tension, nous avions fait l'hypothèse qu'il fallait engager une rechercheaction fondée sur le partenariat ${ }^{1}$ pour produire des connaissances et apprentissages plus adaptés et des changements plus durables (Chia, 2004). C'est ainsi que nous avons expérimenté et formalisé la démarche de Conception en partenariat de systèmes agricoles innovants (CPSAI) dans une optique " pas à pas ».

L'objectif de l'article est de présenter la CPSAI et de l'illustrer par une étude de cas concernant la gestion de la fumure organique dans des exploitations familiales de polyculture-élevage. Nous nous appuierons sur les résultats de dix années de travaux au Burkina Faso au cours desquels les différentes composantes de la CPSAI ont été testées : modalités d'organisation et d'engagement des acteurs (Koutou et Vall, 2010) ; prise en compte des savoirs locaux (Blanchard et al., 2013 ; Vall et Diallo, 2009) ; expérimentation en partenariat chez l'agriculteur (Blanchard et al., 2014 ; Coulibaly et al., 2012) ; introduction de la modélisation dans la conception (Andrieu et al., 2012). Nous aborderons, en discussion, les forces et les limites de la démarche. Enfin nous résumerons la CPSAI en deux principes essentiels.

\section{La démarche CPSAI: explorer, expérimenter, innover}

\subsection{Phase 1 : exploration et formalisation du partenariat}

\subsubsection{Exploration : le temps du diagnostic} et de la problématisation

À ce stade, les agriculteurs ont exprimé leurs préoccupations. L'objectif était alors de jauger et de contextualiser les

\footnotetext{
${ }^{1}$ C'est-à-dire une association d'acteurs d'origines diverses, tant institutionnelle que territoriale, acceptant de mutualiser des efforts et des moyens en vue de réaliser un objectif défini en commun et/ou de résoudre un problème ou un besoin clairement identifié.
}

problèmes. On a réalisé des diagnostics rapides, multi-scalaires et participatifs (Fig. 1) et on a consulté les travaux antérieurs. Le recours à des objets intermédiaires (Jeantet, 1998) a été très utile pour élaborer une représentation commune du problème et envisager des solutions, mais aussi pour faciliter le dialogue des chercheurs avec les acteurs de terrain. Le diagnostic visait à avoir une meilleure compréhension des contraintes, des stratégies et des moyens que les agriculteurs mettaient en œuvre pour résoudre leurs problèmes et à repérer le système et les jeux d'acteurs, la motivation, la représentativité, la légitimité, la compétence des acteurs.

Lors de la restitution du diagnostic, les acteurs de terrain et les chercheurs ont débattu des résultats et se sont construits une représentation commune de la situation initiale et des problèmes à traiter. Ainsi un collectif d'acteurs (CA) composé d'agriculteurs, de conseillers agricoles et de chercheurs a commencé à émerger. Pour aller de l'avant, le CA est entré dans un exercice de problématisation, c'est-à-dire dans la construction d'un raisonnement visant à établir des liens entre les problèmes et leurs causes possibles. Ce travail lui a permis d'élaborer, en salle ou sur le terrain, non seulement des hypothèses de recherche mais aussi une première liste de solutions envisageables. Lorsque le CA estimait qu'il avait les connaissances et les moyens de vérifier les hypothèses formulées, il pouvait décider de se lancer dans la recherche de solutions. Dans le cas contraire, il lui fallait chercher des partenaires plus qualifiés ou des moyens d'action complémentaires.

La problématisation a permis de dégager les premiers éléments de ce que furent les points de passage obligés de la recherche (Callon, 1986) en termes d'acteurs (sur qui s'appuyer pour créer de la confiance et faciliter la communication entre agriculteurs et chercheurs, chez qui expérimenter) et d'activités à mener (exploration, formation, expérimentation, production des références, etc.). Mais ici, l'engagement des acteurs n'était pas encore définitif.

\subsubsection{Formalisation du partenariat : cadre éthique et organes de gouvernance}

Le CA n'était pas « naturellement » organisé pour impulser une dynamique de changement et il dut s'organiser pour répartir les tâches à effectuer (explorer des pistes, débattre des options possibles, en expérimenter certaines, les évaluer). Dès lors, il lui fallut formaliser son organisation et commencer à programmer son action (les objectifs poursuivis, les moyens à mobiliser pour atteindre les objectifs visés et les indicateurs de réalisation). Le consentement mutuel de l'engagement des acteurs du collectif fut scellé dans un contrat écrit ou oral formalisant ainsi le cadre éthique de la CPSAI (engagements réciproques du CA sur les valeurs partagées, les objectifs communément poursuivis, les règles du jeu et les moyens commis). Parallèlement, pour favoriser la participation du plus grand nombre et pour gérer les asymétries entre acteurs, furent mis en place des organes de gouvernance dont le modèle canonique issu de nos travaux comportait trois comités :

- des comités locaux villageois, composés d'agriculteurs et de conseillers agricoles, chargés de l'animation de la CPSAI et de la mise en œuvre de la programmation des activités ;

- un comité de pilotage, composé par les représentants des institutions (recherche, développement, producteurs), qui décidait des orientations stratégiques, validait les 


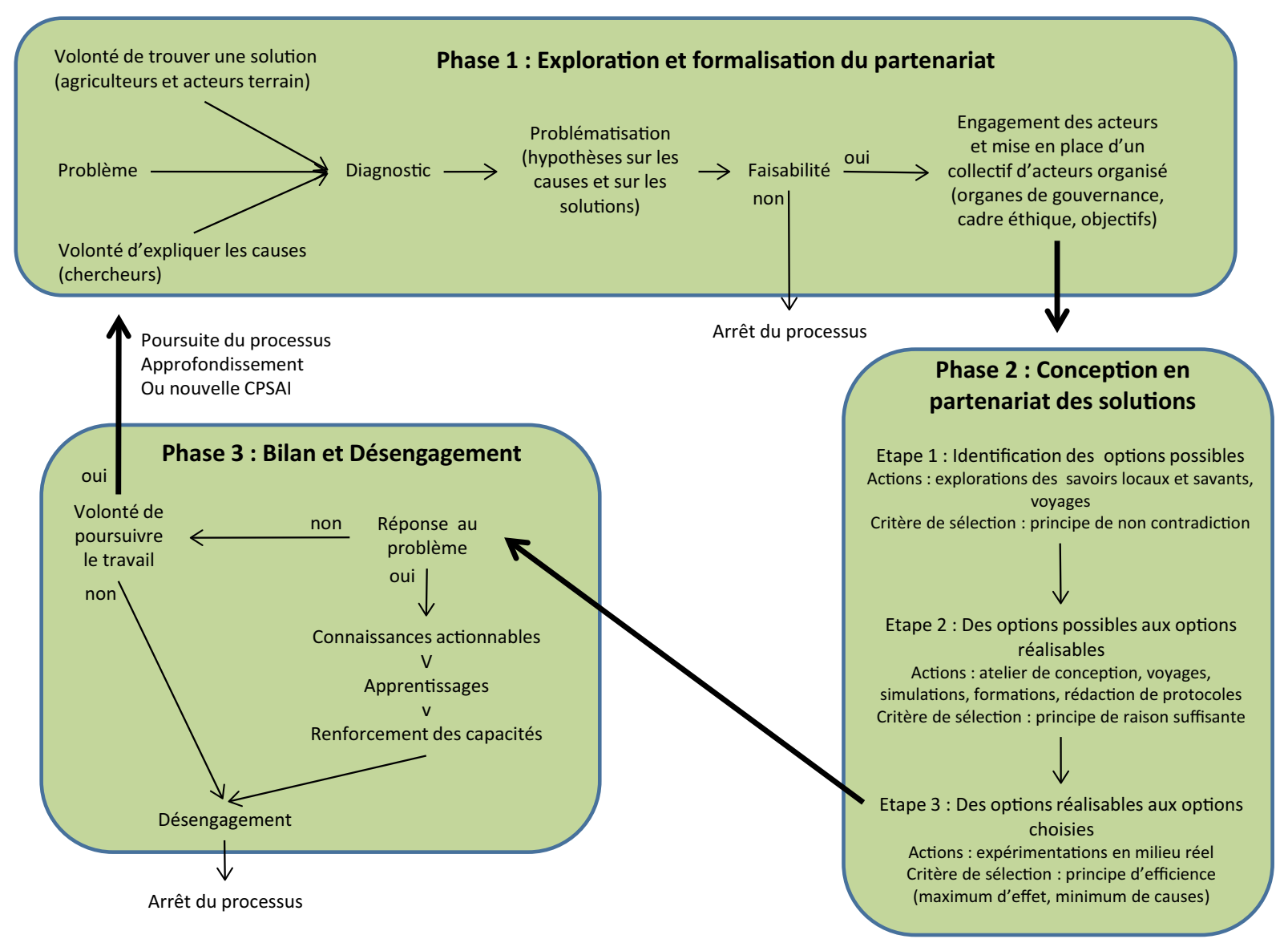

Fig. 1. Schématisation de la conception en partenariat de systèmes agricoles innovants.

Fig. 1. Schematic of participatory process for the design of innovative farming systems.

propositions des comités villageois et la programmation des activités, et arbitrait en cas de conflit ;

- un comité scientifique, composé d'experts reconnus sur la problématique, qui facilitait la distanciation et la réflexibilité méthodologique, proposait des orientations stratégiques au comité de pilotage et accompagnait les chercheurs dans la valorisation des résultats.

La formalisation du partenariat permit de constituer un CA solidaire et responsable, agissant en connaissance de cause et dans le respect des règles établies.

\subsection{Phase 2 : conception en partenariat des innovations}

Ici, le travail du CA a consisté à élaborer des chemins de changement en traitant successivement les questions suivantes :

- Quelles sont les options possibles pour résoudre le problème?

- Quelles sont les options réalisables, c'est-à-dire répondant le mieux aux objectifs et aux contraintes des acteurs ?

- Comment adapter ces options pour optimiser les effets recherchés ?

\subsection{1 Étape 1 : identification des options possibles}

À ce stade, le CA s'interrogeait encore sur les options possibles pour traiter le problème. Il s'engageait alors dans un travail de recensement des options envisageables, et procédait à un inventaire des connaissances scientifiques disponibles, des savoirs et des pratiques locales liées au problème qu'il cherchait à résoudre. Il supposait que les solutions pouvaient provenir tant des avancées scientifiques que des savoirs locaux, et plus probablement d'un travail d'hybridation des savoirs scientifiques et profanes. Dans cet inventaire initial, le CA retenait les options qui lui paraissaient envisageables dans le contexte local, établissant ainsi une liste de solutions possibles.

\subsection{2 Étape 2 : des options possibles aux options réalisables}

Pour identifier les options réalisables (c'est-à-dire celles qui correspondent aux objectifs et aux contraintes des acteurs de terrain), le CA a organisé des voyages d'études pour que les agriculteurs candidats à l'innovation discutent « du pour ou du contre » avec des agriculteurs ayant innové, et de la faisabilité de ces options. Des exercices d'exploration, à l'aide de modèles de fonctionnement des exploitations agricoles, ont été progressivement introduits pour simuler et discuter des 
changements (Andrieu et al., 2012). À partir de ces éléments, le CA sélectionnait une liste restreinte d'options réalisables parmi la liste des options possibles. Au cours de cette étape, les enjeux étaient importants, puisqu'ils concernaient le choix des options à tester et l'identification des producteurs qui seraient chargés de les mettre en œuvre (Coulibaly et al., 2012). Pour éviter les dérives (notamment le favoritisme et les distributions inégales des ressources véhiculées par le CA), le recours au cadre éthique (établit en fin de phase 1) fut parfois nécessaire pour arbitrer. L'élaboration par le CA d'un protocole, spécifiant les engagements réciproques des acteurs sur les opérations à conduire, clôturait cette séquence précédant le passage à l'expérimentation des options réalisables.

\subsection{3 Étape 3 : des options réalisables aux options choisies}

Ici, les options réalisables étaient testées en vraie grandeur, pour être adaptées avant de parvenir aux options choisies par le CA. Ces réalisations se faisaient chez des «producteurs expérimentateurs » et nécessitaient à la fois un suivi des résultats techniques et économiques, mais aussi une attention particulière à la réaction des acteurs de terrain face au principe innovant. Comme l'intervention se déroulait le plus souvent dans un contexte de ressources limitées, l'application du principe d'efficience permettait au CA d'identifier les solutions produisant le maximum d'effet (gain de production et préservation des ressources naturelles, autrement dit un accroissement durable de la production), avec le minimum de causes (intrants de synthèses, travail, capital, aménagement de l'espace). Les options acceptables et efficientes étaient retenues par le $\mathrm{CA}$.

\subsection{Phase 3 : bilan et désengagement}

\subsubsection{Bilan}

L'objectif de la CPSAI a été atteint quand le CA a développé des capacités suffisantes pour apporter des réponses au problème de départ et pour faire face à des situations similaires. La CPSAI a renforcé les capacités du CA à :

- mobiliser des partenaires, forger des alliances face à des problèmes complexes ;

- analyser une situation et anticiper les évolutions possibles ;

- rechercher les causes des problèmes pour agir à leur niveau ;

- formuler des hypothèses sur les solutions possibles aux problèmes ;

- hiérarchiser et trier les options pour favoriser celles que l'on souhaite mettre en œuvre ;

- les adapter pour qu'elles produisent les effets voulus.

Du côté des chercheurs, la CPSAI a renforcé leur capacité à produire des connaissances dans et pour l'action (Sebillotte, 2002).

Ces capacités nouvelles ont été le fruit d'apprentissages techniques et organisationnels (Argyris et Schön, 1978), et de connaissances actionnables (Avenier et Schmitt, 2007) produits par la CPSAI. Ces dernières spécifiaient les moyens et activités à mettre en œuvre pour atteindre les résultats attendus et les conditions techniques et organisationnelles à satisfaire pour que les propositions soient adoptées par les agriculteurs.
L'adoption des principes innovants validait les hypothèses de départ concernant le problème à traiter, et renseignait sur les conditions de validité des solutions proposées. Dans ce cas, le cheminement pouvait être considéré comme valable dans des circonstances analogues, ce qui lui conférait une certaine généricité. Mais la CPSAI aboutissait parfois à une impasse quand certaines contraintes et ressources étaient omises lors du diagnostic, et dans une telle situation, le travail de problématisation était relancé.

\subsubsection{Désengagement}

Tant que les solutions produites ne satisfaisaient pas le CA, et tant que ce dernier persistait dans sa volonté de poursuivre le travail, le cycle de la CPSAI pouvait se poursuivre (Fig. 1). Pourtant, la CPSAI, comme toute recherche-action, a un début et une fin (Chia, 2004 ; Liu, 1997) qu'il faut essayer de préciser dans le contrat initial ( $c f$. phase 1). En théorie, le désengagement ou la dissolution du CA arrive quand, du côté des agriculteurs et des conseillers agricoles, les projets de changements souhaités sont réalisés, que l'on a appris à résoudre les problèmes qui se posaient au départ et, quand, du côté des chercheurs, les propositions explicatives des phénomènes étudiés ont été validées et transformées en proposition d'innovations. Mais dans notre cas, le désengagement a coïncidé avec la fin d'un projet alors que les résultats escomptés n'étaient pas totalement atteints. Des attentes non satisfaites ont certainement généré des frustrations. Mais cela n'a pas conduit à une crise parce que les résultats atteints furent jugés suffisants et parce que lors de la phase d'engagement, le CA avait pris soin d'énoncer clairement les conditions de réalisation de l'action (date de début et de fin, financements disponibles, etc.), de programmer le désengagement en définissant des objectifs réalistes ainsi que des indicateurs d'avancement vérifiables et enfin de doter le comité de pilotage d'une fonction d'arbitrage pour qu'il puisse intervenir en cas de conflit.

\section{La CPSAI à I'œuvre : application au cas des pratiques de gestion des fumures organiques (FO)}

\subsection{Phase 1 : exploration et formalisation du partenariat}

\subsubsection{Exploration}

En 2005, l'un des premiers problèmes soulevé par les producteurs a été la baisse de fertilité des sols et les difficultés à produire de la fumure organique. Le diagnostic initial (Blanchard et al., 2014) a confirmé que la production de FO ne suffisait ni en quantité ( $9 \%$ des besoins théoriques couverts, $33 \%$ des exploitations possédant une fosse fumière à la maison et $7 \%$ une fosse au champ) ni en qualité ( $83 \%$ des FO présentaient des teneurs en carbone et en azote inférieures à $12 \mathrm{~g} \mathrm{C} / 100 \mathrm{~g} \mathrm{MS}$ et $0,6 \mathrm{~g} \mathrm{~N} / 100 \mathrm{~g} \mathrm{MS}$ ).

Les causes ont ensuite été identifiées au cours d'ateliers participatifs : manque d'équipement en fosses et charrettes, lacunes de connaissances pour valoriser les biomasses telles que les tiges de coton, main d'œuvre insuffisante, et enfin un modèle verrouillé sur la fosse fumière à la maison. C'est à partir de là que le CA commença à imaginer un modèle de production 
de la FO nécessitant peu de main d'œuvre et d'intrants, laissant la nature travailler quand c'est possible, délocalisant une partie de la production au champ pour réduire la manutention et le transport, permettant de valoriser les tiges de coton, et décida d'aller de l'avant dans sa conception (Koutou et Vall, 2010).

\subsubsection{Formalisation du partenariat}

Le partenariat s'est cristallisé dans des comités villageois intitulés comités de concertation villageois ( $\mathrm{CCV}$; Koutou et Vall, 2010), composés de représentants des groupements de producteurs du village (cotonniers, éleveurs, etc.), de conseillers agricoles locaux et de l'équipe de chercheurs, tous volontaires. Le CCV intervenait à toutes les étapes de la CPSAI, et plus particulièrement pour la mobilisation des acteurs locaux, l'organisation des rencontres et le choix des expérimentateurs. Il se réunissait régulièrement et tenait une assemblée générale de bilan et de programmation en fin d'année.

Composé d'acteurs peu habitués à travailler ensemble, le bon fonctionnement du CCV s'est avéré fortement lié à la présence d'animateurs capables de traduire le discours des uns dans un langage compréhensible par les autres (Akrich et al., 1988). Assurée au départ par des conseillers agricoles et les chercheurs, l'animation fut progressivement prise en charge par les agriculteurs du bureau exécutif du $\mathrm{CCV}$, ce qui a renforcé le climat de confiance et a facilité la production d'éléments de langage originaux et compréhensibles par tous (association agriculture-élevage $=$ amitié agriculture-élevage). Ces « passeurs de frontières » ont maintenu un lien permanent entre les membres locaux du CCV et les chercheurs.

\subsection{Phase 2 : conception en partenariat des innovations}

\subsection{1 Étape 1 : identification des options possibles}

Le CA a poursuivi l'exploration des options possibles, en dressant un état de l'art des connaissances scientifiques sur la FO en Afrique de l'Ouest (Berger, 1996 ; Dugué, 1999) et un inventaire des savoirs locaux et des pratiques paysannes de gestion de la FO (Blanchard et al., 2013). Il a organisé des voyages d'étude au sud du Mali, où des pratiques innovantes de gestion des FO avaient été repérées, afin de créer des échanges directs entre agriculteurs burkinabés et maliens permettant de discuter de l'intérêt des options et des conditions de leur mise en place. Le produit de cette étape fut une liste étendue d'options possibles (fumier, compost, tas d'ordures, terre de parc, parc à litière, fosse au champ et à la maison, bio-digesteur, fientes de chauves-souris et volailles, hachage, arrosage, activateur biologique...), dans laquelle certaines options jugées peu réalistes furent écartées par le CA (lisier, charbon de bois à usage agricole).

\subsection{2 Étape 2 : des options possibles aux options réalisables}

La considération des contraintes et des priorités des producteurs ont orienté le CA vers la conception d'un modèle de production de FO multi-fosses et à moindre coût (sans apports d'eau artificiels, sans hachage, sans retournement), sans renoncer à la qualité du produit : FO produite « à la maison » à base de déjections et de refus fourragers divers et FO produite « au champ » à base de tiges de coton et d'apports limités de déjections. Ce modèle pouvait être adapté en nombre, en dimension et en nature (fosse de dimension double, adjonction d'un parc ou d'un bio-digesteur...) selon les besoins et possibilités individuels.

\subsection{3 Étape 3 : des options réalisables aux options choisies}

L'adaptation des modes de production de FO « à la maison » et de FO «au champ » s'est faite à travers un important programme d'installation de fosses à FO chez les agriculteurs (au nombre de 1600 entre 2008 et 2012), de suivis de production de FO (98 fosses paysannes suivies de 2008 à 2012) et d'expérimentations en station pour ajuster les techniques de production de FO (effet de l'abandon du hachage et du retournement sur les pertes et les rendements de production). Une méthode d'analyse multivariée des données, relatives aux modes de conduite, a permis de repérer les options les plus efficientes au regard des objectifs visés, et de spécifier avec le $\mathrm{CA}$ les conditions à respecter pour produire une $\mathrm{FO}$ de qualité acceptable (Blanchard et al., 2014).

\subsection{Phase 3 : bilan et désengagement}

Au terme du programme en 2012, le CA avait produit un nouveau référentiel technique de gestion des $\mathrm{FO}$ en fosses multiples et à moindre coût, avec des innovations particulières pour la production au champ (remplissage unique de la fosse en fin de saison sèche de l'année $n$ et vidange en saison sèche $n+1$, utilisation de tiges de coton, élimination du hachage et des retournements, et avec la pluie comme unique apport d'eau), que l'on pouvait apparenter à un corps de connaissances actionnables. Les évaluations de fin de programme réalisées à cette période (données non publiées) ont montré que la majorité des agriculteurs ayant participé à la CPSAI (65\%) maîtrisait ce référentiel technique, contre $33 \%$ sur l'ensemble de la population, et que l'innovation semblait enclenchée puisque $21 \%$ des agriculteurs n'ayant pas participé aux expérimentations avaient commencé à mettre en place des fosses au champ. Les connaissances entraient donc en action, et ces apprentissages commençaient à modifier les pratiques.

Au final, la CPSAI avait contribué à renforcer les capacités des acteurs à répondre au problème de gestion des $\mathrm{FO}$, ce qui se traduisait par un relèvement de la production de $\mathrm{FO}$ en fosses (elle a triplé entre 2008 et 2012 chez les agriculteurs membres de la CPSAI, données non publiées) et par une reformulation des normes d'épandage pour prendre en compte la qualité de la FO et le type de sol (Blanchard et al. [2014] recommandent 2,4 à $5,3 \mathrm{t} \mathrm{MS} / \mathrm{ha} / \mathrm{an}$, alors que les standards paramétrés sur une FO de haute qualité recommandaient 2,0 à 2,5 t MS/ha/an [Berger, 1996]). Dans les exploitations, les impacts des changements de pratiques commençaient à se faire sentir à travers la réduction des temps de travaux de 12 à $9 \mathrm{~h}-\mathrm{j} / \mathrm{t}$ FO et l'amélioration de l'efficience de la production de FO (grâce à la réduction des tâches et au calage du cycle de production sur les saisons).

\section{Discussion : forces et limites de la CPSAl}

La CPSAI dépasse la simple adoption classique puisqu'elle développe les capacités des acteurs à co-construire des 
réponses aux problèmes, via la production de connaissances actionnables (Avenier et Schmitt, 2007) et d'apprentissages (Argyris et Schön, 1978), autrement dit de savoirs et de savoirfaire de « $1^{\text {re }}$ boucle » (modification des pratiques de production et d'application des FO) et de « $2^{\mathrm{e}}$ boucle » (élaboration d'une stratégie d'amélioration de la fertilité du sol par délocalisation de la production de FO au champ et utilisation sélective de la FO), voire même de « $3^{\mathrm{e}}$ boucle » en touchant aux modes d'organisation des acteurs (persistance de certains CCV après la phase projet, permettant la poursuite des apprentissages).

L'évolution du travail en boucles itératives (Fig. 1) donne de la flexibilité au processus de conception, puisque cela permet la révision des objectifs et l'adaptation des méthodes chemin faisant, comme toute démarche participative constructiviste (Anadon, 2007 ; Liu, 1997). Elle incite à élargir le cercle des acteurs pour prendre en compte les causes des problèmes pouvant se situer à d'autres échelles et à ce titre devrait faciliter la mise en place et le travail de plateformes d'innovation (Adekunle, 2009).

$\mathrm{Au}$ cours du processus, les acteurs partagent savoirs profanes et connaissances scientifiques (Blanchard et al., 2013), ce qui permet d'éclairer la rationalité des pratiques locales par la prise en compte des systèmes de classification utilisés au quotidien par les agriculteurs.

L'enchaînement inexorable des phases et des étapes de la démarche, présentée sur la Figure 1, ne doit cependant pas faire illusion. À chaque pas, des interrogations, des transactions, des délais de prises de décision sont nécessaires et confèrent en réalité un caractère tâtonnant à la démarche (« le chemin se fait en marchant »). Réviser les diagnostics, reproblématiser, reconsidérer les engagements, reprendre les étapes de la conception, réévaluer les résultats font partie du déroulement normal d'une CPSAI.

Les coûts de transaction entre partenaires qu'elle requiert et les compromis qu'elle induit peuvent être perçus par les acteurs comme une restriction de leur liberté, qui pour les chercheurs s'ajoutent à la crainte de ne pas pouvoir publier rapidement et en abondance, vu le caractère itératif et tâtonnant de la démarche (Neef et Neubert, 2011).

Le financement de la recherche par projets, dans lesquels on séduit le bailleur de fonds avec un cadre logique ambitieux, tend à verrouiller a priori le choix des options possibles. Lors des phases de soumission d'un projet de CPSAI, la formulation du cadre logique doit être adaptée en conséquence, pour éviter de basculer dans une conception réglée. Quoi qu'il en soit, il est difficile de définir a priori le temps nécessaire pour innover durablement, comme tentent de l'imposer les projets.

La CPSAI butte aussi sur les asymétries entre acteurs, et particulièrement entre agriculteurs et chercheurs, tant sur le plan des ressources matérielles qu'immatérielles. La réduction des asymétries relève de la gageure. Il faut les assumer et tenter de les gérer en partageant l'information avec les partenaires, en respectant leurs droits de parole et de participation aux décisions, en prévoyant un accès équitable aux moyens matériels, etc.

La CPSAI présentée dans cet article correspond à une conception innovante conduite dans une perspective " pas à pas ». Dans une optique de conception « de novo », la place et le rôle des agriculteurs et des conseillers agricoles restent encore à préciser pour qu'ils soient acteurs des exercices de construction de scénarios. Pour identifier les points suscepti- bles de poser problème dans le processus de participation, la grille d'analyse proposée par Gouttenoire et al. (2014) pourrait être utilisée. En pratique, il s'agira de préciser sur quoi les acteurs non-chercheurs s'engageraient si ce n'est pour changer les choses à court terme (comme cela est le cas dans la CPSAI orientée « pas à pas »), et aussi de réfléchir à l'adaptation des modèles de simulation pour que ces derniers soient attrayants pour les acteurs de terrain (des modèles comportant peu de données d'entrée, prenant en compte les catégories locales, proposant des sorties facilement compréhensibles, etc.).

\section{Conclusion}

La CPSAI est née de la rencontre d'acteurs (agriculteurs, conseillers agricoles et chercheurs), souhaitant modifier la réalité. Elle vise à produire des connaissances actionnables et des apprentissages pour que les acteurs soient en capacité de mieux maîtriser l'innovation. Elle se déroule dans le cadre de dispositifs de gouvernance explicites dont les membres se considèrent partenaires et reconnaissent un cadre éthique qu'ils ont eux-mêmes élaboré pour garantir le respect des valeurs et des objectifs négociés. Nous tirons deux leçons principales de ces travaux :

- premièrement, que l'union des acteurs autour d'un intérêt commun (solidarité), en toute connaissance de cause (responsabilité), est fondatrice de l'engagement ;

- deuxièmement, que la construction du changement doit être progressive : explorer d'abord le champ des possibles, ensuite choisir parmi les possibles les options correspondant le mieux aux évolutions voulues, et enfin retenir celles qui maximisent les effets recherchés tout en minimisant les externalités négatives.

Dans une CPSAI, les réponses aux problèmes sont coproduites sur mesure et par conséquent répondent aux attentes des acteurs, mais leur domaine de validité reste localisé. Pour étendre leur rayon d'action, garantir leur durabilité et anticiper leurs impacts, le changement d'échelle et l'élargissement de la compréhension du contexte nous semblent nécessaires, ce qui suggère les pistes de recherche suivantes :

- comment impliquer les acteurs des filières et des territoires dans le dispositif;

- comment coupler la conception avec des dispositifs d'observation des impacts du changement?

\section{Références}

Adekunle AA. 2009. Integrated Agriculture Research for Development (IAR4D). Accra, Ghana: Forum for Agricultural Research in Africa (FARA).

Adekunle AA, Ellis-Jones J, Ajibefun I, et al. 2012. Agricultural innovation in sub-Saharan Africa: experiences from multiplestakeholder approaches. Accra, Ghana: Forum for Agricultural Research in Africa (FARA).

Akrich M, Callon M, Latour B. 1988. À quoi tient le succès des innovations ? Le choix des porte-parole. Gérer et Comprendre. Ann. Mines 12: 4-29.

Anadon M. 2007. La recherche participative. Multiples regards. Quebec: Presse Universitaire de Quebec. 
Andrieu N, Dugué P, Le Gal P Y, Rueff M, Schaller N, Semporé A. 2012. Validating a whole farm modelling with stakeholders: Evidence from a West African case. J. Agri. Sci. 4: 159-173.

Argyris C, Schön DA. 1978. Organizational Learning: A Theory of Action Perspective. Reading, UK: Addison-Wesley MA.

Avenier MJ, Schmitt C. 2007. La Construction de savoirs pour l'action. Paris: L'Harmattan.

Berger M. 1996. L'amélioration de la fumure organique en Afrique soudano-sahélienne. Agriculture et développement (hors-série). Montpellier: Cirad.

Blanchard M, Vayssières J, Dugué P, Vall E. 2013. Local technical knowledge and efficiency of organic fertilizer production in South Mali: Diversity of practices. Agroecol. Sustain. Food Syst. 37: 6672-6699.

Blanchard M, Coulibaly K, Bognini S, Dugué P, Vall E. 2014. Diversité de la qualité des engrais organiques produits par les paysans d'Afrique de l'Ouest: quelles conséquences sur les recommandations de fumure ? Biotechnol. Agron. Soc. Environ. 18: 4.

Callon M. 1986. Éléments pour une sociologie de la traduction. La domestication des coquilles Saint-Jacques et de marins pêcheurs dans la Baie de Saint-Brieuc. Annee Sociol. 36: 169-208.

Chia E. 2004. Principes, méthodes de la recherche en partenariat : une proposition pour la traction animale. Rev. Elevage Med. Vet. Pays Tropicaux 57: 233-240.

Chia E, Dedieu B. 2002. Nouveaux dispositifs de RD en agriculture : le programme franco-argentin «IDEAS ». Cah. Agric. 11: 259-268.

Coulibaly K, Vall E, Autfray P, Bacye B, Nacro HB, Sedogo MP. 2012. Co-conception d'itinéraires techniques de culture pure du niébé (Vignaunguiculata L. Walp.) et du mucuna (Mucunadeeringiana [Bort], Merrill) dans la zone cotonnière ouest du Burkina Faso : intérêts et limites. J. Agric. Environ. Int. Dev. 106-1: 139-155.

Dedieu B, Aubin J, Duteurtre G, Alexandre G, Vayssières J, Bommel P, Faye B. 2011. Conception et évaluation de systèmes d'élevage durables en régions chaudes. INRA Prod. Anim. 24-1: 113-128.

Dugué P. 1999. Utilisation de la biomasse végétale et de la fumure animale : impacts sur l'évolution de la fertilité des terres en zone de savanes. Montpellier: Cirad-Tera.

Gouttenoire L, Taverne M, Cournut S, Hostiou N, Houdart M, Lardon S. 2014. Faciliter les échanges entre chercheurs sur les projets de recherche participative : proposition d'une grille d'analyse. Cah Agric. 23: 205-212.

Jeantet A. 1998. Les objets intermédiaires dans la conception. Éléments pour une sociologie des processus de conception. Sociol. Trav. 3: 291-316.

Koutou M, Vall E. 2010. Implication des acteurs locaux dans la conception d'innovations : le cas des systèmes agropastoraux du Tuy (Burkina-Faso). In: Coudel E, Devautour H, Soulard C, Hubert $\mathrm{B}$, eds. Innovation and sustainable development in agriculture and food. Montpellier: International symposium ISDA 2010, abstracts and papers, pp. 12.

Le Masson P, Weil B, Hatchuel A. 2006. Les processus d'innovation : conception innovante et croissance des entreprises. Paris: Hermes Lavoisier.

Liu M. 1997. Fondements et pratiques de la recherche action. Paris: L'Harmattan.

Meynard JM, Dourmad JY. 2014. L'innovation en élevage. Acquis et fronts de recherche. INRA Prod. Anim. 27-2: 77-88.

Meynard JM, Dedieu B, Bos AP. 2012. Re-design and co-design of farming systems. An overview of methods and practices. In: Darnhofer I, Gibon D, Dedieu B, eds. Farming systems Research into the 21st century: the new dynamic. Springer, pp. 407-432.

Mikolasek O, Barlet B, Chia E, Pouomogne V, Tomedi Eyango Tabi M. 2009. Développement de la petite pisciculture marchande au Cameroun : la recherche-action en partenariat. Cah. Agric. 18: 270-276.

Neef A, Neubert D. 2011. Stakeholder participation in agricultural research projects: a conceptual framework for reflection and decision-making. Agric. Hum. Values 28: 179-194.

Sebillotte M. 2002. Agronomes et territoires. Les trois métiers des agronomes. In : Prévost $\mathrm{P}$, dir. Agronomie et Territoires, deuxième édition des entretiens du Pradel. Paris: L'Harmattan, pp. 479-497.

Vall E, Chia E. 2014. Coconstruire l'innovation : la recherche-action en partenariat. In: Sourrisseau JM, ed. Agricultures familiales et mondes à venir. Versailles: Quae.

Vall E, Diallo M.A. 2009. Savoirs techniques locaux et pratiques : la conduite des troupeaux aux pâturages (Ouest du Burkina Faso). Nat. Sci. Soc. 17-2: 122-135.

Cite this article as: Vall E, Chia E, Blanchard M, Koutou M, Coulibaly K, Andrieu N. 2016. La co-conception en partenariat de systèmes agricoles innovants. Cah. Agric. 25: 15001. 